\title{
Conformal Geometry in Engineering and Medicine
}

\section{Wei Zeng*}

Stony Brook University, Stony Brook, New York 11790, USA

With the development of 3D geometric acquisition technologies, massive $3 \mathrm{D}$ geometric data are ubiquitous today. It is of great challenges to process and analyze 3D geometric data efficiently and accurately. Computational conformal geometry [1] is an emerging inter-disciplinary field, which combines modern geometry with computer science and offers rigorous and practical tools for tackling massive geometric data processing problems. The concepts and methods in conformal geometry play fundamental roles in many fields in engineering and medicine.

Conformal geometry studies the invariants under the conformal transformation (angle preserving mapping) group. Conformal geometry is more flexible than Riemannian geometry, and more rigid than topology. Conformal geometry is capable of unifying all shapes in real world to one of three canonical shapes, the sphere, the plane, or the hyperbolic disk; conformal geometric algorithms convert 3D geometric processing problems to $2 \mathrm{D}$ image processing problems; furthermore, all surfaces in real life have conformal structures, therefore conformal geometric methods are general. These merits make conformal geometry a powerful tool for real applications.

Ricci flow [2] is a fundamental geometric tool for studying geometric structures and modeling the real physical phenomena. It has been successfully applied in the proof of Poincare's conjecture, which deforms the Riemannian metric proportionally to the curvature, such that the curvature evolves according to a heat diffusion process. Ricci flow allows users to design Riemannian metrics using prescribed Gaussian curvatures. Therefore, it can be applied directly for shape registration and geometric analysis. Ricci flow can also be used for computing general surface mappings [3]. In recent years, the Ricci flow theory has been discretized, and applied for 3D human face registration and deformable surface tracking in computer vision; global surface parameterization in computer graphics; conformal brain mapping and virtual colonoscopy in medical imaging; homotopy detection in computational topology; delivery guaranteed greedy routing and load balancing in wireless sensor network, and so on.

Applying modern geometry to practical fields has fundamental importance. The public resources are greatly helpful for wide access to the fast developing technologies. The OMICS’ Applied \& Computational Mathematics Open Access policy (http://www.omicsonline.org/ OpenAccess.php) and the special features provided by the publisher (http://www.omicsonline.org/special-features.php) help the community reach the goal.

\section{References}

1. Gu DX, Zeng W, Luo F, Yau ST (2011) Numerical Computation of Surface Conformal Mappings. CMFT 11: 747-787.

2. Zeng W, Samaras D, Gu DX (2010) Ricci Flow for 3D Shape Analysis. IEEE T Pattern Anal 32: 662-677.

3. Zeng W, Lui ML, Luo F, Chan T, Yau ST, et al. (2012) Computing Quasicon formal Maps on Riemann Surfaces Using Discrete Curvature Flow. Numer Math 1-33.
*Corresponding author: Wei Zeng, Stony Brook University, Stony Brook, New York 11790, USA, E-mail: zengwei@cs.stonybrook.edu

Received April 02, 2012; Accepted April 05, 2012; Published April 09, 2012

Citation: Zeng W (2012) Conformal Geometry in Engineering and Medicine. J Applied Computat Mathemat 1:e105. doi:10.4172/2168-9679.1000e105

Copyright: (c) 2012 Zeng W. This is an open-access article distributed under the terms of the Creative Commons Attribution License, which permits unrestricted use, distribution, and reproduction in any medium, provided the original author and source are credited. 